\title{
INTELIGENCIA EMOCIONAL Y COMPORTAMIENTOS ALIMENTARIOS EN ESTUDIANTES DE LA FACULTAD DE EDUCACIÓN DE LA UNIVERSIDAD DE ZARAGOZA
}

\author{
Eva Urbón Ladrero \\ Departamento de Psicología y Sociología \\ Universidad de Zaragoza \\ eurbon@unizar.es
}

Recepción Artículo: 22 octubre 2021 Admisión Evaluación: 22 octubre 2021 Informe Evaluador 1: 23 octubre 2021 Informe Evaluador 2: 24 octubre 2021 Aprobación Publicación: 24 octubre 2021

\section{RESUMEN}

Esta investigación se llevó a cabo en la Facultad de Educación de la Universidad de Zaragoza. Los objetivos del estudio fueron caracterizar el comportamiento alimentario de la muestra, en función del sexo y analizar la posible relación de estas variables con los comportamientos alimentarios y la inteligencia emocional.

La muestra estuvo compuesta por 115 alumnos pertenecientes al Grado de Magisterio de Educación Primaria y al Master del Profesorado de Secundaria.

La hipótesis de partida fue que aquellas personas con puntuaciones más bajas en inteligencia emocional, presentaban comportamientos alimentarios más desadaptados, que podían influir en su IMC. También se analizaron las horas de deporte y de sueño, así como la calidad de este, con el fin de comprobar si dichas variables podían estar relacionadas con la inteligencia emocional.

Los comportamientos alimentarios, el sueño y la actividad física se analizaron a través de un cuestionario diseñado para tal fin. La inteligencia emocional se estudió por medio de las respuestas obtenidas en la escala de inteligencia emocional EIS.

Palabras clave: inteligencia emocional; comportamientos alimentarios; IMC; deporte y sueño

\section{ABSTRACT}

Emotional intelligence and eating behaviors in students of the faculty of education of the university of Zaragoza. This research was carried out at the Faculty of Education of the University of Zaragoza. The objectives of the study were to characterize the eating behavior of the sample, based on sex and to analyze the possible relationship of these variables with abnormal eating behaviors and emotional intelligence. 


\section{INTELIGENCIA EMOCIONAL Y COMPORTAMIENTOS ALIMENTARIOS EN ESTUDIANTES DE LA FACULTAD DE EDUCACIÓN DE LA UNIVERSIDAD DE ZARAGOZA}

The sample consisted of 115 students belonging to the Primary Education Teaching Degree and the Secondary Teaching Master's Degree.

The starting hypothesis was that those people with lower scores in emotional intelligence presented more maladaptive eating behaviors, which could influence their BMI. The hours of sport and sleep, as well as the quality of this, were also analyzed in order to check whether these variables could be related to emotional intelligence.

Eating behaviors, sleep and physical activity were analyzed through a questionnaire designed for this purpose. Emotional intelligence was studied through the responses obtained on the Schutte (1998) EIS emotional intelligence scale.

The results obtained showed that the students with the highest scores in El were the ones who presented the most dysfunctional eating behaviors, in addition to the fact that these were related to higher values in BMI, less practice of sports and poorer quality and quantity of sleep

Keywords: emotional intelligence; eating behavior; BMl; sport and sleep

\section{INTRODUCCIÓN}

Qué alimentos seleccionamos, cómo, cuándo, dónde y con quién nos alimentamos, tiene consecuencias patológicas físicas y mentales. Vivimos en una sociedad sobrealimentada, con tendencias encaminadas a mal nutrirnos (Penrose, 2007) y en la que se oferta conocimiento y se comunica ignorancia, pero cuyas decisiones se toman desde la inteligencia emocional (Millán, 2000). Nuestras creencias median, entre otras cosas en la elección de alimentos, "cuando el ser humano tiene la probabilidad de elegir, opta por unos alimentos que simbólicamente se traducen en unos valores que encajan con su forma de entender la realidad. ... dentro de unos límites materiales y simbólicos modelados por la cultura" Cantarero (2002, p. 175).

Son numerosas las investigaciones que asocian trastornos mentales con ingesta alimentaria determinada y comportamientos alimentarios disfuncionales. Los estados biológico y psicológico de las personas influyen en la alimentación de las mismas (Apfeldorfer, 1995), así personas que presentan patologías relacionadas con la ansiedad y la depresión, activan distintas estructuras cerebrales que determinan la elección de determinados alimentos y predisponen a que tengan comportamiento alimentarios más desadaptados, concretamente manifestados con una alimentación restringida y emocional (Almajwal, 2016; Neparevá, Hosu, Kuosma, Ukkola, Uitti, y Laitinen, 2012) El apetito es un estado mental, modificable y susceptible a las distintas situaciones emocionales, en el momento en que nuestras células no reciben los aportes nutritivos adecuados, la persona sufre una importante carga de estrés debida a una demanda interna insatisfecha (Díaz Franco, 2007).

Existe también una relación entre alimentación e inteligencia emocional (I.E.) (Watson, 1979). Las personas con déficit en inteligencia emocional presentan mayor predisposición a tener estrés, ansiedad, depresión y problemas interpersonales; en cambio un alto coeficiente emocional se asocia con una buena salud física y mental (Extremera y Fernández-Berrocal, 2005). Del mismo modo que la nutrición altera la I. E., la I. E. alterada puede inducir a patologías nutricionales (Contreras y Gracia, 2005). Determinados comportamientos alimentarios desadaptados e ingestas alimentarias particulares, seguidos por personas que padecen trastornos de ansiedad 0 depresión, inhiben la producción de endorfinas, las cuales refuerzan dichos comportamientos, agravando seriamente su salud (Margules, 1978). La inteligencia emocional disfuncional, altera la nutrición; hasta tal punto que personas que siguen dieta y no regulan adecuadamente sus emociones, presentan una ingesta poco saludable de alimentos. Las personas con dificultades emocionales puede manifestar problemas con la alimentación y desarrollar conductas alimentarias desadaptativas como el hambre voraz o la inhibición de la ingesta (Abramson, 1999). Existe una ingesta emocional causada por hipersensibilidad, ansiedad, depresión (Ganley, 1989)

Las personas que reconocen darse atracones, reconocen que gestionan mal sus emociones, además de estar más predispuestas a experimentar ansiedad o depresión durante la ingesta, especialmente si esta es excesiva (Linswiller, Crowther y Stephens, 1989)

Cruz, Pascual, Echeburúa y Etxebarría (2013) enfatizan que es necesario entrenar a la población en riesgo de presentar trastornos de la conducta alimentaria, en técnicas de expresión y regulación emocional para que no ten- 
gan que recurrir a conductas inadecuadas de control del peso y de la imagen corporal. Esta población mostró que la insatisfacción corporal se asociaba significativamente a menor frecuencia de emociones positivas, mayor frecuencia de emociones negativas, percepción más negativa de las emociones, actitud más negativa hacia la expresión emocional, mayor grado de alexitimia y mayor utilización de formas de afrontamiento disfuncionales.

Por otra parte, distintas investigaciones muestran la relación entre inteligencia emocional y sobrepeso u obesidad. La inteligencia emocional está relacionada con el estado anímico en los pacientes con obesidad (Barroso, 2018). El estrés está relacionado con el nivel de inteligencia emocional, porcentaje de grasa e IMC (Arce, 2018). Los sujetos con sobrepeso u obesidad presentaron bajo cociente emocional, lo que indicaría que perciben cierto grado de malestar psicológico, como así también expresaron baja capacidad intrapersonal y dificultad de manejo del estrés y adaptabilidad. Sin embargo manifestaron buenas habilidades interpersonales y demostraron adecuado estado de ánimo (Salafia, Casari y Vera, 2015).

La calidad de sueño y el deporte son variables que afectan a la I.E. La falta de sueño puede afectar seriamente a nuestra capacidad para regular las reacciones emocionales (Beattie, Kyle, Colin y Viello, 2015). Por último, el deporte es una manera de reducir el estrés y afrontar de forma saludable distintas situaciones estresantes. (Lopes, Côté y Salovey, 2014).

\section{OBJETIVOS DE LA INVESTIGACIÓN}

Analizar si determinados comportamientos alimentarios guardan relación con el IMC, el sueño y el deporte. Averiguar si existe relación entre los comportamientos alimentarios y la inteligencia emocional.

Comprobar la relación entre inteligencia emocional e IMC.

\section{MUESTRA}

La muestra de la investigación, estuvo conformada por 114 alumnos de la Facultad de Educación de la Universidad de Zaragoza.

Los criterios de inclusión fueron: cursar en la actualidad una titulación universitaria en la Facultad de Educación y cumplimentar la totalidad del cuestionario. Participaron 127 alumnos, de los que finalmente quedaron excluidos13 por no rellenar adecuadamente los informes.

En la tabla 1 se exponen los datos descriptivos de la muestra, Ilamando la atención que el 12,28\% del alumnado presenta obesidad o sobrepeso y un $11,4 \%$ infrapeso. El $73,68 \%$ de la muestra es mujer. La edad media es de 26,15 años.

Tabla 1

Datos descriptivos de la muestra

\begin{tabular}{|c|c|c|c|}
\hline VARIABLES & $\begin{array}{l}\text { MUJERES } \\
(n=84)\end{array}$ & $\begin{array}{l}\text { HOMBRES } \\
(n=30)\end{array}$ & $\begin{array}{l}\text { TOTAL } \\
(n=114)\end{array}$ \\
\hline Edad* & $25,96(7,74)$ & $26,34(4,75)$ & $26,15(7,08)$ \\
\hline $\operatorname{IMC}\left(\mathrm{Kg} / \mathrm{m}^{2}\right)^{*}$ & $21,17(3,74)$ & $22,78(3,01)^{*}$ & $21,59(3,62)$ \\
\hline Obesidad & $2(2,38)$ & $1(3,33)$ & $3(2,63)$ \\
\hline Sobrepeso & $7(8,33)$ & $3(10)$ & $11(9,65)$ \\
\hline Normopeso & $64(76,19)$ & $24(80)$ & $88(77,19)$ \\
\hline Infrapeso & $11(13,1)$ & $2(6,67)$ & $13(11,4)$ \\
\hline $\begin{array}{l}\text { Estudios en } \\
\text { curso** } \\
\quad \text { Magisterio }\end{array}$ & $38(45,24)$ & $28\left(93,33^{)}\right.$ & $66(57,89)$ \\
\hline $\begin{array}{l}\text { Infantil } \\
\text { Máster } \\
\text { Profesorado }\end{array}$ & $46(54,76)$ & $2(6,67)$ & $48(42,11)$ \\
\hline
\end{tabular}

Nota: *Celdas representan medias y desviaciones típicas**Celdas representan frecuencias y porcentajes respecto al mismo grupo. Obesidad (IMC $\geq 30$ ), Sobrepeso (IMC $\geq 25)$, Normopeso $(18,6 \mathrm{~kg} / \mathrm{m} 2 \leq \mathrm{IMC} \leq 24,99 \mathrm{~kg} / \mathrm{m} 2)$ e infrapeso (IMC $\leq 18,5$ $\mathrm{kg} / \mathrm{m} 2)$. 


\section{INTELIGENCIA EMOCIONAL Y COMPORTAMIENTOS ALIMENTARIOS EN ESTUDIANTES DE LA FACULTAD DE EDUCACIÓN DE LA UNIVERSIDAD DE ZARAGOZA}

\section{METODOLOGÍA Y/O INSTRUMENTOS UTILIZADOS}

La metodología que se empleo fue la siguiente: se diseñó un cuestionario en google forms que pretendía dar respuesta a los objetivos del estudio, una vez comprobada su viabilidad se informó a los profesores de la Facultad del estudio a llevar a cabo y la finalidad de este y se instó a los mismos a que animaran a participar al alumnado de sus clases. El período de recogida de los cuestionarios fue de dos semanas.

Para el análisis de los instrumentos de evaluación, se trabajó con el paquete informático SPSS 20 para Windows (Norusis, 2011).

En todas las pruebas estadísticas se estableció como nivel de significación bilateral $p<0,05$. Así mismo se estableció como prueba para contrastar la normalidad del conjunto de datos el Test de Kolmogorov-Smirnov. Al no darse una distribución normal en algunas variables, fue necesario hacer un ajuste de las puntuaciones obtenidas en dichas variables por logaritmos.

Para averiguar la relación entre comportamientos alimentarios, IMC, deporte y sueño, se realizaron correlaciones de Pearson. Para observar si existían diferencias significativas en comportamientos alimentarios y sexo, se utilizó el estadístico Chi cuadrado. Para comprobar la diferencia de medias entre los factores de ElS y el sexo o comportamientos alimentarios se aplicó la prueba t de Student. La prueba ANOVA de un factor se trató para averiguar diferencias significativas entre los cuatro grupos de IMC (infrapeso, normopeso, sobrepeso y obesidad). Por último, para poder determinar qué variables independientes podían estar más relacionadas con la inteligencia emocional de los estudiantes, se realizaron análisis de regresión lineal múltiple.

En relación a los instrumentos utilizados, se diseñó un cuestionario ad hoc para evaluar distintas características de los participantes, entre las que destacaban aquellas sociodemográficas, tales como, edad, sexo, peso, talla, kilos incrementados en los últimos meses, hábitos alimentarios (comidas que realizaba al día, si se saltaba comidas, si picaba entre horas, si comía rápido, etcétera) y de salud, tales como consumo, horas de sueño, calidad de este y ejercicio físico, etc. El cuestionario estaba compuesto por 35 preguntas, y aunque se formulaban preguntas abiertas y cerradas, la mayor parte del mismo presentaba un formato de respuesta de alternativas múltiples. Dentro de la misma aplicación de google forms se pasó a la muestra la Escala de Inteligencia Emocional (EIS, Schutte et al., 1998), traducido y validado al castellano (Salavera y Usán, 2019) Este cuestionario se compone de 33 items, las respuestas son tipo Likert de 5 puntos y van desde 1 (totalmente en desacuerdo) hasta 5 (totalmente de acuerdo). Las escalas y los ítems que se analizan están basadas en el modelo de I.E. de Mayer, Salovey y Caruso (2000), son las siguientes:

Atención a las propias emociones. Items: 6,7, 8, 9,19 y 22.

Atención a las emociones de los demás. de los demás. Items: 5,18, 29 y 32.

Regulación de las propias emociones. Items: 2, 3, 10, 12, 14, 23 y 28.

Expresión emocional. Items: 1, 15, 16, 25 y 33.

Regulación emocional en los otros. Items: 4, 11, 13, 24, 26 y 30.

Emociones en la resolución de conflictos. Items: 17, 20, 21, 27 y 31.

\section{RESULTADOS ALCANZADOS}

Los comportamientos alimentarios más desadaptados se presentaron en el grupo de hombres, los cuales reconocieron: ingerir grandes cantidades de comida $\left({ }^{2}=17,04 ; p=0,000\right) .(O R=0,14)$, repetir plato $\left({ }^{2}=33,99\right.$; $p=0,000)$, $(O R=0,04)$, Ilenarse mucho el plato ( $\left.{ }^{2}=19,22 ; p=0,000\right)$. $(0 R=0,14)$, Ilenarse mucho la boca $\left({ }^{2}=12,75\right.$; $p=0,000),(0 R=0,19)$ y esconder comida $\left({ }^{2}=5,29 ; p=0,02\right),(O R=0,10)$. 
Tabla 2

Comportamientos alimentarios presentados por sexo

\begin{tabular}{|c|c|c|c|c|}
\hline & $\begin{array}{c}\text { MUJERES } \\
(n=84)\end{array}$ & $\begin{array}{c}\text { HOMBRES } \\
(n=30)\end{array}$ & $\begin{array}{c}O R \\
(95 \% I C)\end{array}$ & $\begin{array}{l}\text { TOTAL } \\
(n=114)\end{array}$ \\
\hline $\begin{array}{l}\text { Preocupación por el } \\
\text { peso }\end{array}$ & $26(30,95)$ & $11(36,67)$ & NS & $37(32,46)$ \\
\hline Saltos comidas & $37(44,05)$ & $11(36,67)$ & NS & $48(42,11)$ \\
\hline Horarios regulares & $5(5,95)$ & $5(16,67)$ & NS & $10(8,77)$ \\
\hline Comida casera & $1(1,19)$ & $0(0)$ & NS & $1(0,88)$ \\
\hline Dieta & $10(11,9)$ & $4(13,33)$ & NS & $14(12,28)$ \\
\hline Picar & $44(52,38)$ & $19(63,33)$ & NS & $63(55,26)$ \\
\hline Comer rápido & $35(41,67)$ & $10(33,33)$ & NS & $45(39,47)$ \\
\hline Comer lento & $22(26,19)$ & $16(53,33)$ & NS & $38(33,33)$ \\
\hline Comida rápida & $12(14,26)$ & $4(13,33)$ & NS & $16(14,04)$ \\
\hline Grandes cantidades & $10(11,9)$ & ${ }_{(46,67)^{* * * k}}$ & $\begin{array}{l}0,14(0,05- \\
0,38)\end{array}$ & $24(21,05)$ \\
\hline Viendo TV & $54(64,29)$ & $18(60)$ & NS & $72(63,16)$ \\
\hline Repetir plato & $4(4,76)$ & $15(50)^{* * *}$ & $\begin{array}{l}0,04(0,01- \\
0,16)\end{array}$ & $19(16,67)$ \\
\hline No dejar nada en plato & $53(63,1)$ & $9(30)$ & NS & $62(54,39)$ \\
\hline Llenar mucho el plato & $20(23,81)$ & $20(66,67)^{* * *}$ & $\begin{array}{l}0,14(0,05- \\
0,35)\end{array}$ & $40(35,09)$ \\
\hline Masticar poco & $34(40,48)$ & $11(36,67)$ & NS & $45(39,47)$ \\
\hline Llenarse la boca mucho & $13(15,48)$ & $\begin{array}{c}14 \\
(46,67)^{* * * * k}\end{array}$ & $\begin{array}{l}0,19(0,07- \\
0,5)\end{array}$ & $27(23,68)$ \\
\hline $\begin{array}{l}\text { Tragar antes de otro } \\
\text { bocado }\end{array}$ & $5(5,95)$ & $3(10)$ & NS & $8(7,02)$ \\
\hline Comer por la calle & $13(15,48)$ & $3(10)$ & NS & $16(14,04)$ \\
\hline Comer hasta llenarte & $30(35,71)$ & $15(50)$ & NS & $45(39,47)$ \\
\hline Atracones & $12(14,29)$ & $6(20)$ & NS & $18(15,79)$ \\
\hline Comer por ansiedad & $27(32,14)$ & $9(30)$ & NS & $36(31,58)$ \\
\hline Esconder comida & $1(1,19)$ & $3(10)^{*}$ & $0,10(0,10-1)$ & $4(3,51)$ \\
\hline $\begin{array}{l}\text { Enfadarse si racionan } \\
\text { comida }\end{array}$ & $7(8,33)$ & $3(10)$ & NS & $10(8,77)$ \\
\hline
\end{tabular}

Nota: *Celdas representan medias y desviaciones típicas**Celdas representan frecuencias y porcentajes respecto al mismo grupo. OR: Odds ratio. IC: $95 \%$ Intervalo de Confianza: ${ }^{*} \mathrm{p}<0,01,{ }^{*} \mathrm{p}<0,05$; NS: valor de $\mathrm{p}$ no significativo

El grupo de mujeres fue el que presentó puntuaciones más altas en la puntuación total del cuestionario $(t=2,07, p=0,04)$, así como en determinados factores de la escala EIS, concretamente en la regulación emocional interpersonal $(t=2,14, p=0,03)$ y la resolución de problemas $(t=3,01, p=0,003)$. 
Tabla 3

Factores escala EIS por sexo

\begin{tabular}{lll}
\hline VARIABLES & $\begin{array}{l}\text { MUJERES } \\
(n=84)\end{array}$ & $\begin{array}{l}\text { HOMBRES } \\
(n=30)\end{array}$ \\
\hline APE & $2,40(1,48)$ & $2,93(1,27)$ \\
\hline AED & $3,99(0,55)$ & $3,91(0,49)$ \\
\hline AUTO & $3,31(0,49)$ & $3,35(0,36)$ \\
\hline EE & $1,57(0,11)$ & $1,65(0,10)$ \\
\hline REO & $\mathbf{3 , 6 5 ( 0 , 4 6 ) *}$ & $3,42(0,62)$ \\
\hline RPP & $\mathbf{4 , 1 2 ( 0 , 5 2 ) ^ { * * }}$ & $3,77(0,57)$ \\
\hline TOTAL & $\mathbf{1 2 6 , 4 2 ( 1 2 , 3 7})^{*}$ & $120,96(11,76)$ \\
\hline
\end{tabular}

Nota: *Celdas representan medias y desviaciones típicas. APE: atención a las propias emociones, AED: atención a las emociones de los demás, AUTO: autorregulación, EE: expresión emocional, REO: regulación emociones otros, RPP: resolución de problemas. Las celdas representan las medias y las desviaciones típicas por cada grupo, IC: $95 \%$ Intervalo de confianza: ${ }^{* * *} \mathrm{p}<0,001 ; * * \mathrm{p}<0,01 ; * \mathrm{p}<0,05$; NS: No significativo; $\mathrm{t}=\mathrm{val}$ ar $\mathrm{t}$ de Student

En relación a los resultados que se encontraron entre los distintos comportamientos alimentarios y el IMC, deporte y sueño (véase tabla 4), las correlaciones más significativas se dan entre el hábito de saltarse comidas y menor tiempo de deporte $(r=-0,25, p=0,002)$, la preocupación por el peso y el poco cansancio al levantarse $(r=-$ $0,47, p=0,003)$, el picar entre horas y menor tiempo de deporte $(r=-0,31, p=0,002)$, el Ilenarse mucho la boca mientras se come y un IMC más elevado $(r=0,27, p=0,004)$, y el consumo de comida casera y dormir menos horas $(r=-0,27, p=0,002)$.

Los sujetos de la muestra que se comportaban de forma anómala en su alimentación, tuvieron problemas de peso, practicaban menos deporte, y la calidad y cantidad de sueño se mostraba disfuncional.

Tabla 4

Correlaciones entre comportamientos alimentarios, IMC, deporte y sueño

\begin{tabular}{|c|c|c|c|c|c|}
\hline $\begin{array}{l}\text { Comportamientos } \\
\text { alimentarios }\end{array}$ & $I M C$ & Deporte & $\begin{array}{l}\text { Horas de } \\
\text { sueño }\end{array}$ & $\begin{array}{l}\text { Calidad } \\
\text { de sueño }\end{array}$ & $\begin{array}{c}\text { Sentirse } \\
\text { cansado al } \\
\text { levantarse }\end{array}$ \\
\hline Preocupación por el peso & $r=0,24^{*}$ & $r=-0,19^{*}$ & NS & NS & $r=-0,47 * *$ \\
\hline Saltos de comidas & NS & $\begin{array}{c}r=- \\
0,25 * *\end{array}$ & NS & NS & NS \\
\hline Comida casera & NS & NS & $r=-0,27 * *$ & $\begin{array}{c}r=- \\
0,22 *\end{array}$ & $r=-0,22 *$ \\
\hline Dieta & $r=0,20^{*}$ & NS & NS & NS & NS \\
\hline Picar entre horas & NS & $\begin{array}{c}r=- \\
0,31 * *\end{array}$ & NS & NS & NS \\
\hline Comer rápido & $r=0,18^{*}$ & NS & NS & NS & NS \\
\hline Comida rápida & NS & $\begin{array}{c}r=- \\
0,24 * *\end{array}$ & $r=-0,19^{*}$ & NS & NS \\
\hline No dejar nada en el plato & NS & NS & NS & $\begin{array}{c}r=- \\
0,19^{*}\end{array}$ & $r=-0,19^{*}$ \\
\hline Masticar poco la comida & $r=0,20^{*}$ & NS & NS & NS & NS \\
\hline Llenar mucho la boca & $r=0,27 * *$ & NS & NS & NS & NS \\
\hline $\begin{array}{l}\text { Enfadarse si racionan } \\
\text { comida }\end{array}$ & NS & NS & $r=-0,19^{*}$ & NS & NS \\
\hline
\end{tabular}

Nota: IC: $95 \%$ Intervalo de confianza: ${ }^{* * *} \mathrm{p}<0,001,{ }^{* *} \mathrm{p}<0,01,{ }^{*} \mathrm{p}<0,05$; NS: correlación no significativa 
A la hora de analizar la relación de las escalas de EIS con los comportamientos que presentaron los estudiantes (véase tabla 5), se observó que los estudiantes más preocupados por el peso era los que más atendían sus emociones $(t=-2,26 ; p=0,02)$, gestionaban mejor la resolución de problemas $(t=3,41 ; p=0,001) ; y$ regulaban más adecuadamente las emociones de otros $(t=-1,48 ; p=0,04)$. Aquellos que durante las comidas veían la TV, prestaban más atención a las emociones de los demás $(t=2,99 ; p=0,03)$, se regulaban y expresaban mejor emocionalmente $(t=3,62 ; p=0,000),(t=2,69 ; p=0,008)$ y resolvían problemas con mayor facilidad $(t=-2,20 ; p=0,03)$. Los que repetían plato y comían por la calle, obtenían altas puntuaciones en regulación emocional $(t=2,52$; $p=0,01)$ y $(t=2,22 ; p=0,03)$ respectivamente. Por último, aquellos estudiantes que se enfadaban cuando se les racionaba la comida, eran los que reportaban puntuaciones más elevadas en atención a las propias emociones $(t=2,36 ; p=0,03)$ y resolución de problemas $(t=-2,36 ; p=0,03)$.

Tabla 5

Prueba t escalas EIS según comportamientos alimentarios

\begin{tabular}{|c|c|c|c|c|c|}
\hline $\begin{array}{l}\text { Escalas } \\
\text { EIS }\end{array}$ & $\begin{array}{c}\text { Preocupació } \\
\text { n por el peso } \\
\quad(n=37)\end{array}$ & $\begin{array}{l}\text { Ver Tv } \\
\text { mientras } \\
\text { come } \\
(n=41)\end{array}$ & $\begin{array}{l}\text { Repetir } \\
\text { plato } \\
(n=19)\end{array}$ & $\begin{array}{c}\text { Comer por } \\
\text { la calle } \\
\text { (n=16) }\end{array}$ & $\begin{array}{c}\text { Enfadarse } \\
\text { porque le } \\
\text { racionan } \\
\text { comida }(n=10)\end{array}$ \\
\hline$\overline{\mathrm{APE}}$ & 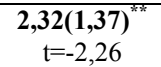 & NS & NS & NS & $\begin{array}{c}\mathbf{3 , 7 0}(\mathbf{0 , 4 8})^{k, k} \\
\mathrm{t}=2,36\end{array}$ \\
\hline AED & NS & $\begin{array}{c}\mathbf{4 , 1 6}(\mathbf{0}, \mathbf{4 8})^{* *} \\
\mathrm{t}=2,99\end{array}$ & NS & NS & NS \\
\hline AUTO & NS & $\underset{*}{3,52(0,48)^{* *}}$ & NS & NS & NS \\
\hline $\mathrm{EE}$ & NS & $\begin{array}{c}\mathrm{t}=3,62 \\
\mathbf{3 , 9 2 ( 0 , 3 9 )} \\
\mathrm{t}=2,69\end{array}$ & NS & NS & NS \\
\hline REO & $\begin{array}{c}3,70(0,48){ }^{* *} \\
t=3,41\end{array}$ & NS & $\begin{array}{c}\mathbf{3 , 2 9}\left(\mathbf{0}, 49^{)^{*}}\right. \\
\mathrm{t}=2.52\end{array}$ & 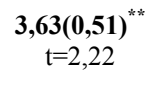 & NS \\
\hline RPP & $\begin{array}{c}\mathbf{4 , 1 0}(\mathbf{0 , 4 8})^{* * *} \\
\mathrm{t}=-1,98\end{array}$ & $\begin{array}{c}\mathbf{4 , 1 8}(\mathbf{0 , 5 5})^{* *} \\
t=-2,20\end{array}$ & NS & NS & $\begin{array}{c}\mathbf{4 , 0 6}(\mathbf{0 , 5 6})^{* * *} \\
\mathrm{t}=-2,36\end{array}$ \\
\hline
\end{tabular}

atención a las propias emociones, demás, AUTO: autorregulación, EE: expresión emocional, REO: regulación emociones otros, RPP: resolución de problemas. Las celdas representan las medias y las desviaciones típicas por cada grupo, IC: $95 \%$ Intervalo de confianza: ${ }^{* * *} p<0,001$; ${ }^{* *} p<0,01 ;{ }^{*} p<0,05$; NS: No significativo; $\mathrm{t}=$ valor $\mathrm{t}$ de Student

Con el fin de analizar diferencias significativas entre grupos de IMC y escalas de EIS, se observó que las únicas diferencias existentes entre las escalas de EIS, se daban en el factor de resolución de problemas donde, las personas con menor peso obtenían medias más altas ( $F=2,93, p=0,03$ ).

Por último, para poder determinar qué variables dependientes podían estar más relacionadas con la puntuación total de la escala EIS, se realizó un análisis de regresión logística múltiple.

Los coeficientes asociados a las variables independientes fueron: edad $(p=0,03)$, sexo $(p=0,16)$, horas de sueño $(p=0,15)$, calidad del sueño $(p=0,000)$, hacer dieta $(p=0,04)$, comer viendo la Tv $(p=0,001)$ y esconder comida $(p=0,007)$.

La probabilidad de tener un mayor coeficiente emocional quedaría determinada por la siguiente ecuación:

Logit $(p)=146,20+0,37$ edad $-8,75$ sexo $-10,19$ horas de sueño $+9,38$ calidad de sueño $-8,3$ hacer dieta -9,51 comer viendo TV - 19,51 esconder comida

La tabla 6, nos presenta el valor explicativo que posee cada variable en relación a la variable dependiente (EIS). A mayor edad, ser mujer, dormir menos horas de sueño, pero de buena calidad y comportamientos ali- 


\section{INTELIGENCIA EMOCIONAL Y COMPORTAMIENTOS ALIMENTARIOS EN ESTUDIANTES DE LA FACULTAD DE EDUCACIÓN DE LA UNIVERSIDAD DE ZARAGOZA}

mentarios de: no seguir dieta, no comer viendo la TV y no esconder comida, tendrían un mayor peso en la puntuación total del coeficiente emocional.

Tabla 6

Prueba de regresión lineal múltiple en relación al coeficiente emocional

\begin{tabular}{lccc}
\hline $\begin{array}{l}\text { Variables } \\
\text { independientes }\end{array}$ & $\begin{array}{c}\text { Coeficien } \\
\text { te Beta }\end{array}$ & $\begin{array}{c}\text { Significació } \\
n \\
\text { estadistica }\end{array}$ & $\begin{array}{c}\text { Error } \\
\text { estánda } \\
r\end{array}$ \\
\hline $\begin{array}{l}\text { Constante } \\
\text { Edad }\end{array}$ & 146,2 & & \\
$\begin{array}{l}\text { Sexo } \\
\text { Horas de }\end{array}$ & 0,37 & 0,03 & 0,17 \\
sueño & $-8,75$ & 0,01 & 3,56 \\
Calidad de & $-10,19$ & 0,01 & 4,90 \\
$\begin{array}{l}\text { sueño } \\
\text { Hacer dieta }\end{array}$ & 9,38 & 0,00 & 2,44 \\
$\begin{array}{l}\text { Comer viendo } \\
\text { TV }\end{array}$ & $-8,30$ & 0,04 & 4,16 \\
$\begin{array}{l}\text { Esconder } \\
\text { comida }\end{array}$ & $-19,51$ & 0,001 & 2,69 \\
\hline
\end{tabular}

\section{DISCUSIÓN}

Los objetivos del estudio fueron caracterizar el comportamiento alimentario de una muestra de estudiantes de la Facultad de Educación y estudiar la relación de dicho comportamiento con el IMC, la práctica de deporte, el sueño y los factores de la escala EIS de inteligencia emocional.

Dentro de la muestra se observó que el grupo de hombres tenía comportamientos alimentarios más desadaptados, pero eran las mujeres las que mostraban tener mejores competencias en gestión emocional. Dichos resultados irían en la línea de la teoría de Millán (2000) que determina que las decisiones alimentarias se toman desde la inteligencia emocional, y de Oda et al. (2015), donde los hombres muestran más conductas alimentarias vinculadas a la sobreingesta y las mujeres realizaban más conductas relacionadas con la restricción alimentaria y la ingesta emocional.

En el presente estudio resultó que determinados comportamientos alimentarios estaban muy relacionados no solo con el peso, también con la práctica de deporte, las horas de sueño o la calidad de este, pero no con los factores analizados de IE, según sucedía en las investigaciones de Beattie et al (2015) 0 Lopes et al. (2014). Los resultados de este estudio parecen indicar que los pensamientos erróneos de los participantes fomentan un estilo de vida poco saludable y siguen comportamientos alimentarios más desadaptados.

Por otra parte, se encontraron relaciones muy significativas entre la inteligencia emocional y determinados comportamientos alimentarios. Dichos datos irían en la línea de los estudios de Díaz Franco (2007), de Watson (1979) que considera el apetito como un estado mental susceptible de distintas situaciones emocionales y de Contreras y Gracia (2005) que reconocen la relación de la alimentación con la inteligencia emocional.

A pesar de que en esta investigación no se encontró una relación entre dieta y regulación emocional (Abramson, 1999) 0 atracones y peor gestión emocional (Linswiller, et. al, 1989); el IMC (Barroso, 2018; Arce, 2018) o los comportamientos alimentarios como la preocupación por el peso, comer mientras se ve la TV, repetir plato, comer por la calle o enfadarse al racionarles la comida, sí que estaban relacionados con la IE. Los sujetos de la muestra podían desarrollar distintas dimensiones de la inteligencia emocional en función de los estilos alimentarios que seguían; por ejemplo, repetir plato o comer por la calle 0 incluso la preocupación por el peso, son comportamientos alimentarios que se hacen en presencia de otros o que se tiene presente el juicio del otro, lo que daría lugar a que estos alumnos fueran más competentes en gestión emocional interpersonal. Del mismo modo, aquellos que respondían con enfados cuando les racionaban la comida o que repetían plato, parece que 
mostraban una gestión emocional basada en su propia experiencia; ya que es habitual que aquellos a los que les racionan la comida sean más conscientes de sus propias emociones o que resuelvan mejor los problemas; así mismo, los participantes que ven la TV mientras comen, serán más atentos con las emociones de los demás, se autocontrolarán mejor, sabrán resolver de manera más satisfactoria los problemas y se expresarán mejor emocionalmente; ya que ver la TV puede ejercer en ellos una influencia positiva en la gestión de las emociones a través del aprendizaje observacional.

En relación al IMC y las competencias emocionales, los estudiantes que pesaban menos, mostraron mayores habilidades emocionales en cuanto a la resolución de problemas, en la investigación de Salafia et al. (2015), Ios IMC por encima del normopeso tenían buenas habilidades interpersonales.

Por último, al tratar de predecir la fórmula que diese con puntuaciones mas altas en IE, se llego a la conclusión que tener más edad, ser mujer, llevar una adecuada higiene de sueño y no seguir determinados comportamientos alimentarios como: seguir dieta (que coincidiría con los resultados del estudio de Abramson, 1999), no comer viendo la TV y no esconder comida, serían los ingredientes adecuados para una adecuada gestión de las emociones.

\section{CONCLUSIONES}

Para poder establecer con mayor rigurosidad conclusiones sobre la relación existente entre el IMC y la IE, se debería haber tenido una muestra más homogénea en cuanto a las cuatro categorías de IMC y sexo.

En posteriores investigaciones sería interesante analizar los comportamientos alimentarios con población clínica diagnosticada de trastornos de ansiedad y depresión, así como estudiar a la población en riesgo de sobrepeso; para corroborar las distintas teorías analizadas que explican la relación de estados emocionales e ingesta y comportamientos alimentarios.

Aún así, los datos obtenidos además de obtener relaciones interesantes entre las variables a estudio, abren la vía para seguir investigando la relación entre la IE y los comportamientos alimentarios, sobretodo teniendo en cuenta otra población distinta a la de la muestra a estudio.

Lo que si que parece evidente es que hay personas que tienen problemas de alimentación que no gestionan bien sus emociones y es necesario apostar por la formación en educación emocional.

Una adecuada gestión de las emociones fomenta hábitos saludables y previene problemas de alimentación disfuncionales, relacionados sobretodo con el control del peso y la imagen corporal.

Esta formación en competencias emocionales debería hacerse extensible a toda población con el fin de prevenir trastornos del comportamiento alimentario, obesidad y otras enfermedades, pero sobretodo para fomentar la salud física y mental.

\section{REFERENCIAS BIBLIOGRÁFICAS}

Abramson, E. (1999). El comer emocional. Bilbao: Desclée de Brouwer. (En el original:

Emocional Eating: a practical Guide to taking control. Lexington Books. 1993).

Almajwal, A (2016). Stress, shift duty, and eating behavior among nurses in Central Saudi Arabia. Saudi Medical Journal, 37(2):191-198

Arce Varela, E. (2018) Relación del nivel de actividad física, IMC, Porcentaje de grasa e inteligencia emocional con el nivel de estrés académico en mujeres Universitarias.

https://repositorio.una.ac.cr/bitstream/handle/11056/11442/Tesis\%209788.pdf?sequence=5\&isAllowed=y

Barroso, K. (2018) Estado de ánimo e inteligencia emocional en pacientes con obesidad. http://hdl.handle.net/10366/139078

Beattie, L., Kyle, S.D., Colin, A.E., Viello, S.M. Social interactions, emotion and sleep: a sistematic review and research agenda. Sleep Medicine Reviews 24(2015) 83-100

Contreras, J. (2002). Los aspectos culturales del consumo de carne. Somos lo que comemos. Gracia Arnáiz, M. (Coord.). Barcelona: Ariel. 
Díaz Franco, J.J. (2007). Estrés alimentario y salud laboral vs. estrés laboral y alimentación equilibrada. Medicina seguridad trabajo. 53(209):93-99.

Extremera, N. y Fernández Berrocal, P. (2005). Perceived emotional intelligence and life satisfaction: Predictive and incremental validity using the Trait Meta-mood scale. Personality and Individual Differences, 39, 937948.

Ganley, R.M. (1989). Emotion and eating in obesity: A review of the literature. International Journal of Eating Disorders, 8.

García Ibáñez, F. (2016) "la influencia de la nutrición y las relaciones sociales" https://riuma.uma.es/xmlui/bitstream/handle/10630/13286/TD_GARCIA_IBANEZ_Filadelfo.pdf?sequence=1\&isAllowed=y

Lingswiler, J.H. y otros. (1987). Emotional reactivity and eating in binge eating and obesity. Journal of Behavioral Medicine, 10

Lopes, P., Côté, S.y Salovey, P. (2014). Un modelo de habilidad de la inteligencia emocional: implicaciones para la evaluación y el entrenamiento en J. Mestre Navas y P. Fernández-Berrocal, Manual de inteligencia emocional (217-234). Madrid: Editorial Pirámide.

Margules, B. (1978). Beta-Endorphin Is Associated with Overeating in Genetically Obese Mice and Rats. Science, 202 (diciembre).

Mayer, J. D., Salovey, P., y Caruso, D. (2000). Models of emotional intelligence. En Handbook of intelligence (pp. 396-420). New York: Cambridge.

Neparevá, N., Hosu, L., Kuosma, E., Ukkola, 0., Uitti, J. y Laitinen, J. (2012). Occupational burnout, eating behavior, and weight among working women. The American Journal of Clinical Nutrition. Abril 2012, 95(4):934943.

Norusis, M. J. (2011). SPSS/PC advanced statistics, v20, software de computadora en disco. Chicago: SPSS Inc

Oda, C., Saldaña, C. y Andrés, A. (2015). Comportamientos alimentarios cotidianos y anómalos en una muestra comunitaria de adultos chilenos. Nutrición Hospitalaria ;32(2):590-599

Pascual, A., Etxebarria, I., Cruz, M.S. y Echeburúa, E. (2011). Las variables emocionales como factores de riesgo de los trastornos alimentarios. International Journal of Clinical and Health Psychology, 11(2), 229-247.

Salafia, Verónica \& Casari, Leandro \& Vera, Micaela. (2015). La inteligencia emocional en personas con sobrepeso y obesidad. Revista Eureka 12. 193-204

Salavera, C., y Usán, P. (2019). Exploración de la dimensionalidad y las propiedades psicométricas de la escala EIS de inteligencia emocional. Rev. CES Psico, 12(3), 50-66

Schutte, N.S., Malouff, J.M., Hall, L.E., Haggerty, D.J., Cooper, J.T., Golden, C.J., \& Dornheim, L. (1998). Development and validation of a measure of emotional intelligence. Personality and Individual Differences, $25,167-177$

Watson, G. (1979). Journal of the Nutricional Academy, II (II) 